\title{
Effects of electroconvulsive therapy and repetitive transcranial magnetic stimulation on serum brain-derived neurotrophic factor levels in patients with depression
}

\author{
Laura Gedge ${ }^{1,2}$, Ashley Beaudoin ${ }^{1}$, Lauren Lazowski ${ }^{1}$, Regina du Toit ${ }^{3}$, Ruzica Jokic ${ }^{3,4}$ and Roumen Milev ${ }^{3,4}$ * \\ Centre for Neuroscience Studies, Queen's University, Kingston, ON, Canada \\ ${ }^{2}$ College of Medicine, Western University of Health Sciences, Pomona, CA, USA \\ ${ }^{3}$ Department of Psychiatry, Queen's University, Kingston, ON, Canada \\ ${ }^{4}$ Providence Care-Mental Health Services, Kingston, ON, Canada
}

Edited by:

Mark A. Frye, Mayo Clinic, USA

Reviewed by:

Peter Kirsch, Zentralinstitut für Seelische Gesundheit, Germany

Khemraj Hirani, University of Miami

Miller School of Medicine, USA

*Correspondence:

Roumen Milev, Department of Psychiatry, Providence Care-Mental Health Services, Queen's University, 752 King Street West, Postal Bag 603, Kingston, ON, Canada K7L 4X3. e-mail: roumen.milev@queensu.ca

\begin{abstract}
Objective: Brain-derived neurotrophic factor (BDNF) levels are decreased in individuals with depression and increase following antidepressant treatment. The objective of this study is to compare pre- and post-treatment serum BDNF levels in patients with drugresistant major depressive disorder (MDD) who received either electroconvulsive therapy (ECT) or repetitive transcranial magnetic stimulation (rTMS). It is hypothesized that non-pharmacological treatments also increase serum BDNF levels. Methods: This was a prospective, single-blind study comparing pre- and post-treatment serum BDNF levels of 29 patients with drug-resistant MDD who received ECT or rTMS treatment. Serum BDNF levels were measured 1 week prior to and 1 week after treatment using the sandwich ELISA technique. Depression severity was measured 1 week before and 1 week after treatment using the Hamilton Depression Rating Scale. Two-sided normal distribution paired $t$-test analysis was used to compare pre- and post-treatment BDNF concentration and illness severity. Bivariate correlations using Pearson's coefficient assessed the relationship between post-treatment BDNF levels and post-treatment depression severity. Results: There was no significant difference in serum BDNF levels before and after ECT, although concentrations tended to increase from a baseline mean of $9.95-12.29 \mathrm{ng} / \mathrm{ml}$ after treatment $(p=0.137)$. Treatment with $r$ TMS did not significantly alter BDNF concentrations $(p=0.282)$. Depression severity significantly decreased following both ECT $(p=0.003)$ and rTMS $(p<0.001)$. Post-treatment BDNF concentration was not significantly correlated with post-treatment depression severity in patients who received either ECT $(r=-0.133$, $p=0.697)$ or rTMS $(r=0.374, p=0.126)$. It is important to note that these results are based on the small number of patients included in this study. Conclusion: This study suggests that ECT and rTMS may not exert their clinical effects by altering serum BDNF levels in patients with drug-resistant MDD. Serum BDNF concentration may not be a biomarker of ECT or rTMS treatment response.
\end{abstract}

Keywords: brain-derived neurotrophic factor, electroconvulsive therapy, repetitive transcranial magnetic stimulation, depression

\section{INTRODUCTION}

Depression is associated with neuronal atrophy and cell loss in discrete regions of the brain, including the prefrontal cortex and the hippocampus (Sapolsky, 1996; Sheline et al., 1996; Sheline et al., 1999; Bremner et al., 2000; Warner-Schmidt and Duman, 2006). Neurotrophins have a crucial role in brain development, survival and maintenance of neuronal functions, and synaptic plasticity (Kaplan and Miller, 2000). The neurotrophin brain-derived neurotrophic factor (BDNF) promotes the survival of central nervous system (CNS) neurons including hippocampal, cortical, cholinergic, nigral dopaminergic, and serotonergic neurons (Alderson et al., 1990; Hyman et al., 1991; Ghosh et al., 1994; Eaton and Whittemore, 1996; Lindholm et al., 1996). Recent research suggests that BDNF is involved in the pathophysiology of depression as well as in the mechanism of action of antidepressant treatments (Hashimoto et al., 2004; Angelucci et al., 2005; Russo-Neustadt and Chen, 2005; Schmidt and Duman, 2010). Serum BDNF levels in antidepressant-naive individuals suffering from major depressive disorder (MDD) tend to be significantly lower than BDNF levels in both individuals treated for depression and healthy controls (Karege et al., 2002a, 2005; Shimizu et al., 2003; Lee et al., 2007; Sen et al., 2008; Bocchio-Chiavetto et al., 2010). Furthermore, BDNF levels are negatively correlated with depression severity.

Pharmacological treatments have been shown to effect serum BDNF levels. While BDNF levels are lower in depressed patients, serum BDNF levels of depressed patients increase after treatment with antidepressants or mood stabilizers (Chen et al., 2001; Shimizu et al., 2003; Aydemir et al., 2005; Gervasoni et al., 2005; 
Gonul et al., 2005; Huang et al., 2007; Yoshimura et al., 2007; Brunoni et al., 2008; Hellweg et al., 2008; Monteleone et al., 2008; Piccinni et al., 2008; Sen et al., 2008; Matrisciano et al., 2009). A recent meta-analysis showed that changes in BDNF levels are significantly correlated with changes in depression severity (Brunoni et al., 2008). The antidepressants sertraline, amitriptyline, paroxetine, and fluoxetine have been particularly successful in raising BDNF levels in patients suffering from MDD (Hashimoto et al., 2004; Aydemir et al., 2005; Yoshimura et al., 2007; Matrisciano et al., 2009).

The effect of non-pharmacological treatments for MDD on serum BDNF levels is conflicting and remains to be established. Both electroconvulsive therapy (ECT) and repetitive transcranial magnetic stimulation (rTMS) are effective in the treatment of MDD and are becoming more widely used as alternatives to pharmacological treatments in drug-resistant patients (Hoffman and Cavus, 2002; Tamminga et al., 2002; Gershon et al., 2003; Schlaepfer et al., 2003; Lisanby, 2007). Researchers have recently investigated the effect of ECT and rTMS on serum BDNF levels. Some studies have shown that ECT increases serum BDNF levels in animals (Angelucci et al., 2002; Altar et al., 2003; Sartorius et al., 2009), as well as in patients with treatment-resistant MDD (Bocchio-Chiavetto et al., 2006; Marano et al., 2007; Okamoto et al., 2008; Hu et al., 2010). Some studies, however, have not been able to replicate this finding (Fernandes et al., 2009; Gronli et al., 2009). Similarly, studies have reported that rTMS increases serum BDNF levels (Yukimasa et al., 2006; Zanardini et al., 2006), while another found that rTMS did not alter serum BDNF levels in patients with MDD (Lang et al., 2006). Therefore, further research is needed to determine the effect of ECT and rTMS on BDNF levels in depressed patients.

The primary objective of this study is to examine the effect of ECT and rTMS on serum BDNF levels in patients with treatmentresistant MDD. Secondly, the correlation between serum BDNF level and illness severity will be assessed. We hypothesize that ECT and rTMS will increase serum BDNF levels, inducing a neuroprotective effect, which contributes to decreased illness severity. A correlation between the change in BDNF levels and change in symptom severity may suggest that BDNF has a central role in the molecular mechanism of action of ECT and rTMS treatments. Furthermore, such a finding would support BDNF as a biochemical marker of MDD.

\section{MATERIALS AND METHODS STUDY DESIGN}

This was a prospective, single-blind study comparing pre- and post-treatment serum BDNF levels of patients with treatmentresistant MDD who were currently experiencing a major depressive episode and received ECT or rTMS treatment. Patients were recruited from a single-center, tertiary care mood disorders clinic. The study was approved by the Queen's University Health Sciences and Affiliated Teaching Hospitals Research Ethics Boards.

\section{STUDY POPULATION}

Patients were recruited from inpatient and outpatient services for the evaluation and treatment of depression. All participants were 18 years of age or older and gave written informed consent. All patients had a Diagnostic and Statistical Manual of Mental Disorders, 4th edition (DSM-IV) diagnosis of MDD, and were currently experiencing a major depressive episode [Hamilton Depression Rating Scale-17 item (HDRS-17) $\geq 15$, Young Mania Rating Scale $($ YMRS $) \leq 8$ ]. Participants with anorexia nervosa, bulimia nervosa, a previous diagnosis of schizophrenia, and/or current substance abuse (except caffeine and nicotine) were excluded. Patients who received either ECT or rTMS within the year prior to entry into the study were excluded. Patients with any other primary psychiatric diagnosis, an uncontrolled medical illness, a severe personality disorder, epilepsy, or any medical illness thought to affect serum levels of BDNF were also excluded from the study. Changes to baseline medication regimes were not allowed in the 3weeks prior to participation or during the study. Study participants were withdrawn from the study if they experienced significant side effects, became pregnant or withdrew consent.

Thirty-one patients were originally enrolled in the trial; however two patients (one in ECT group and one in rTMS group) were excluded due to significant changes in medication over the course of the study. This left 29 patients remaining in the study. Eleven patients received ECT and 18 patients received rTMS. The ECT population consisted of four men and seven women, with a mean age of 46.5 years. The rTMS population consisted of 5 men and 13 women, with a mean age of 44.7 years. Twenty-two of 29 patients were taking antidepressants (escitalopram, sertraline, amitriptyline, venlafaxine, bupropion, citalopram, mirtazapine, fluoxetine, and fluvoxamine), 8 were taking benzodiazepines (clonazepam, lorazepam, temazepam, and oxazepam), and 15 were taking antipsychotics (quetiapine, ziprasidone, olanzapine, and risperidone). Of the 29 patients in the study, 15 were taking a combination of at least two different medications, and the remaining 14 were only taking one medication.

\section{TREATMENTS}

Patients received either ECT or rTMS as a part of their clinical care recommended by a physician. Both treatments were administered as per hospital protocol and under instructions of the treating psychiatrist. ECT was administered over 12 sessions, lasting approximately 4 weeks, with patients receiving an average of 3 sessions per week. rTMS was administered over 10 consecutive daily sessions, lasting approximately 2 weeks. Prior to the start of rTMS treatment, individual motor threshold (MT) on motor cortex was determined following international standard protocol (Rossini et al., 1994). The stimulus intensity was set to $80 \%$ of the patient's MT intensity, with $10 \mathrm{~Hz}$ stimulation of the left dorsolateral prefrontal cortex (DLPFC).

\section{CLINICAL ASSESSMENTS}

Patients were assessed at two time points: baseline ( 7 days before treatment) and 7 days after completing ECT or rTMS treatment. Each clinical assessment consisted of the Hamilton Depression Rating Scale (HDRS).

\section{SERUM BDNF DETERMINATION}

Venous blood samples were collected 7 days prior to treatment and 7 days after treatment completion. Samples were collected in anticoagulant-free tubes. Serum samples were transported on ice 
and allowed to sit at room temperature for $30 \mathrm{~min}$ before separation of the serum by centrifugation. Blood samples were centrifuged at $3000 \mathrm{rpm}$ for $15 \mathrm{~min}$ at $4^{\circ} \mathrm{C}$; serum was drawn off and frozen at $-20^{\circ} \mathrm{C}$ until needed. Serum BDNF levels were measured by a sandwich ELSIA method using the BDNF $E_{\max }$ ImmunoAssay System (Promega, 2009). The manufacturer's instructions were followed.

\section{STATISTICAL ANALYSIS}

Statistical analysis of data was done on SPSS 18.0 for PC (SPSS Inc, Chicago, IL, usa). Two-sided normal distribution paired $t$-test analysis with a significance of 0.05 was conducted to compare preand post-treatment measures. Bivariate correlations were assessed using Pearson's coefficient. A one-way ANOVA with a significance of 0.05 was used to analyze pre-treatment variance in clinical measures and BDNF levels within the study population.

\section{RESULTS}

A repeated measures ANOVA with medication as a covariate was conducted to determine if varying medications throughout the duration of the study had a possible confounding effect on patients' HDRS score and BDNF level outcomes. Nonsignificant results were found for both the HDRS score outcomes, $F(1,20)=1.866, p=0.187$, as well as the BDNF level outcomes, $F(1,20)=1.783, p=0.197$.

\section{ELECTROCONVULSIVE THERAPY}

Baseline BDNF concentrations or HDRS scores did not significantly differ between patients. Mean $( \pm$ SE) baseline serum BDNF concentration was $9.95 \mathrm{ng} / \mathrm{ml}( \pm 1.94)$ and mean post-treatment concentration was $12.29 \mathrm{ng} / \mathrm{ml}( \pm 1.05)$. There was no significant difference between pre- and post-treatment serum BDNF levels $(t=-1.615, \mathrm{df}=10, p=0.137$; Figure 1$)$. Illness severity significantly decreased following ECT $(t=3.955, \mathrm{df}=10, p=0.003)$. Mean $( \pm$ SE) pre-treatment HDRS score was $23.73( \pm 1.43)$ and mean post-treatment HDRS score was 13.27 ( \pm 1.90 ; Figure 2). BDNF concentration was not significantly correlated with HDRS score following ECT $(r=-0.133, p=0.697)$.

\section{REPETITIVE TRANSCRANIAL MAGNETIC STIMULATION}

Baseline BDNF concentrations or HDRS scores did not significantly differ between patients. Mean $( \pm$ SE) baseline serum BDNF concentration was $10.05 \mathrm{ng} / \mathrm{ml}( \pm 1.21)$ and mean post-treatment concentration was $8.90 \mathrm{ng} / \mathrm{ml}( \pm 1.02)$. There was no significant difference between pre- and post-treatment serum BDNF levels $(t=1.111, \mathrm{df}=17, p=0.282$; Figure 3$)$. Illness severity significantly decreased following rTMS $(t=5.645, \mathrm{df}=17, p<0.001)$. Mean $( \pm S E)$ pre-treatment HDRS score was $23.22( \pm 1.15)$ and mean post-treatment HDRS score was 15.11 ( \pm 1.74 ; Figure 4). BDNF concentration was not significantly correlated with HDRS score following rTMS $(r=0.374, p=0.126)$.

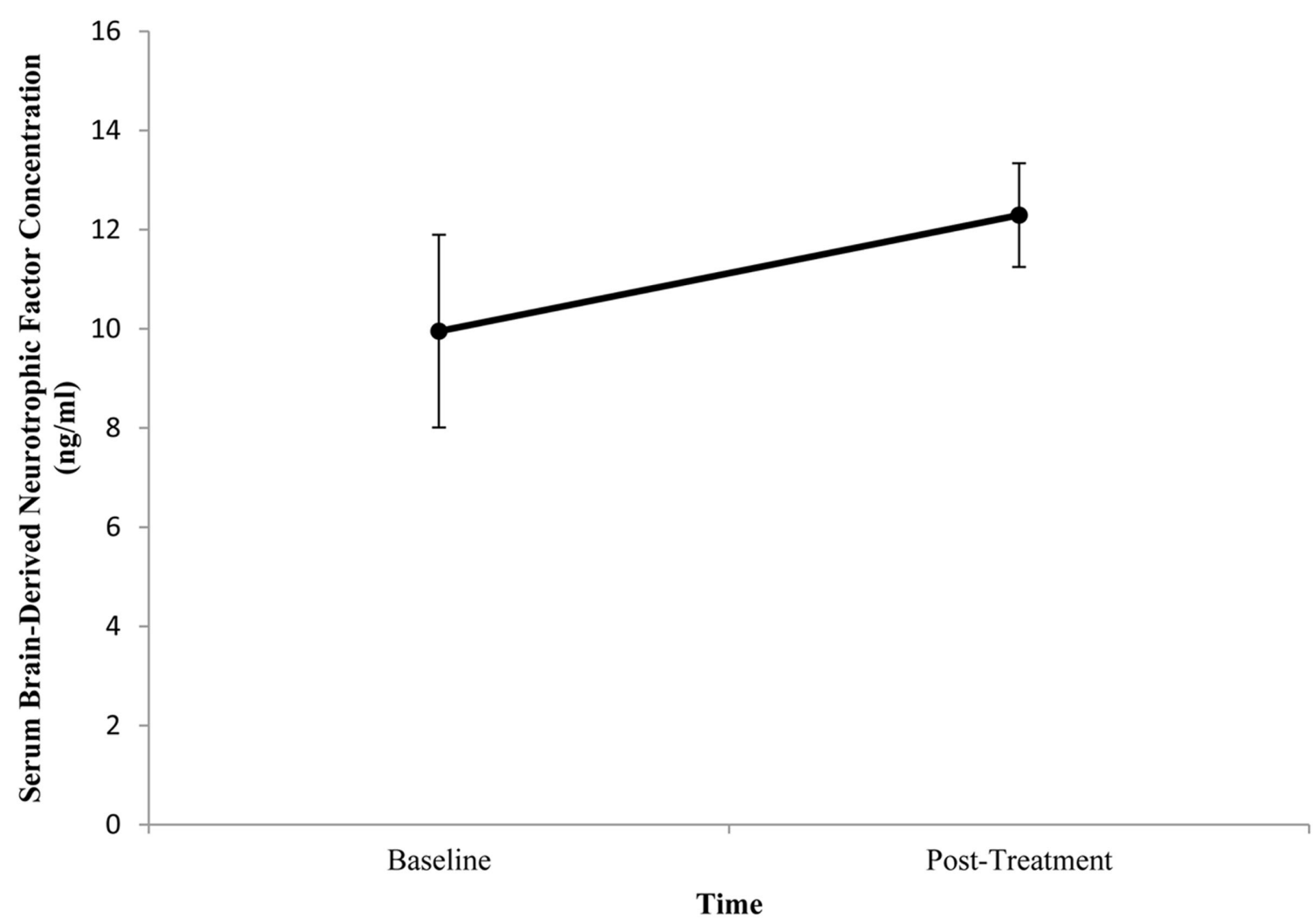

FIGURE 1 | Change in serum brain-derived neurotrophic factor (BDNF) concentration from pre- to post-treatment in patients with major depressive disorder treated with electroconvulsive therapy (ECT). Serum

BDNF was determined 1 week prior to and 1 week following ECT. There was no significant difference between pre- and post-treatment BDNF levels $(p=0.137)$. Values are mean \pm SE. 


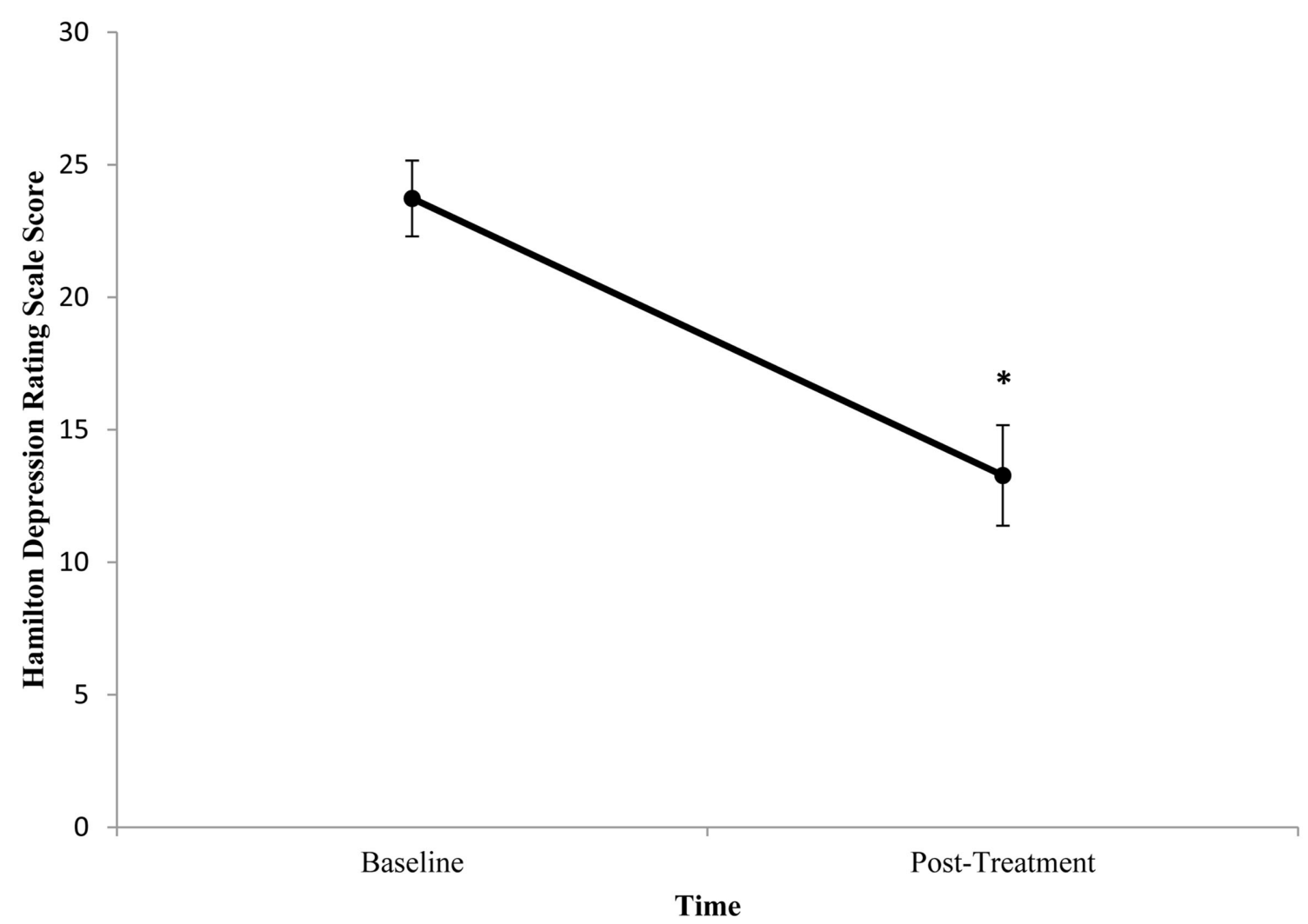

FIGURE 2 | Change in Hamilton Depression Rating Scale (HDRS) score from pre- to post-treatment in patients with major depressive disorder treated with electroconvulsive therapy (ECT).
HDRS scores were determined 1 week prior to and 1 week following ECT. HDRS scores significantly decreased following ECT ( $p=0.003$ ). Values are mean \pm SE.

\section{DISCUSSION}

Neither ECT nor rTMS altered serum BDNF levels of patients with MDD. Depression severity significantly decreased following both ECT and rTMS. The change in serum BDNF levels was not correlated with the change in illness severity.

Several studies have examined the effect of ECT on serum BDNF levels in patients with MDD. Our results were consistent with those of two recent studies that also found that ECT does not alter serum BDNF levels, despite decreasing depression severity (Fernandes et al., 2009; Gronli et al., 2009). This suggests that ECT may not share a similar mechanism of action as antidepressants in improving illness severity in patients with treatment-resistant depression.

However, the results of this study conflict with other studies investigating the effect of ECT on serum BDNF levels. While the current study shows that ECT did not alter BDNF levels, others have reported an increase in serum BDNF levels following ECT in patients with MDD (Marano et al., 2007). However, the timepoint at which post-treatment BDNF levels are measured is not consistent across previous studies. Serum BDNF levels have been measured during treatment (Marano et al., 2007), 5 weeks after the start of ECT (Okamoto et al., 2008), as well as 1 week (Piccini et al., 2009), 2 weeks (Hu et al., 2010), or 1 month (Bocchio-Chiavetto et al., 2006) after treatment completion. Therefore, while ECT may increase BDNF levels, it remains unclear as to when this increase occurs. In this study, BDNF was measured 1 week after treatment completion. Our non-significant findings conflict with those of Piccini et al. (2009) who found that serum BDNF levels are increased 1 week after the completion of ECT. In contrast, Bocchio-Chiavetto et al. (2006) found that BDNF levels were not altered 1 week after ECT, however BDNF levels increased 1 month after treatment compared to pre-treatment levels. These results together may suggest that there is individual variation in the length of time required before neuronal changes can be observed. It is possible that changes in BDNF levels did occur in patients in this study, however it was too early to observe these changes 1 week after treatment completion. More time may have been needed to observe a significant increase in serum BDNF levels from baseline levels.

Few studies have investigated the effect of rTMS on serum BDNF levels in patients with MDD. Contrary to our results, rTMS has been reported to increase BDNF levels in drug-resistant depressed patients (Yukimasa et al., 2006; Zanardini et al., 2006). However, our findings agree with those of Lang et al. (2006) who also found that rTMS does not alter BDNF levels. As the results of this study add to the inconsistency of research findings in this area, the effect of rTMS on BDNF levels in patients with depression remains to be established. 


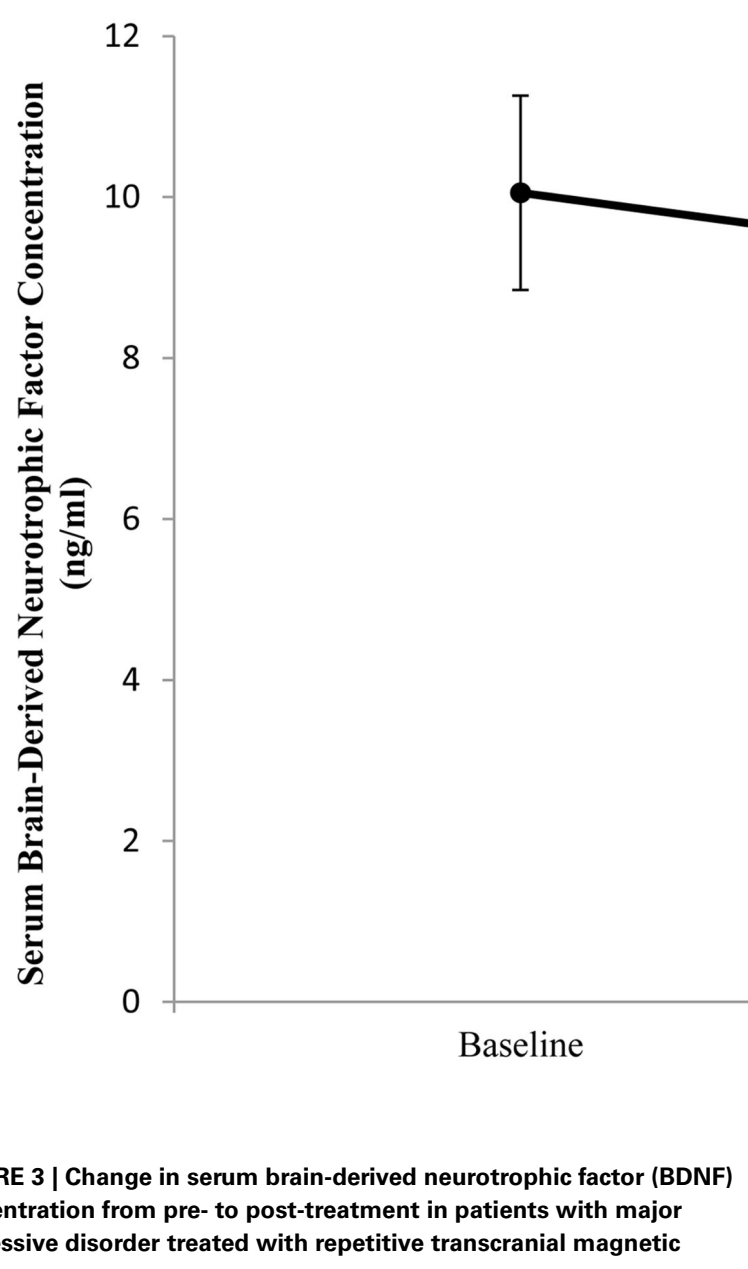

The main limitation in this study is its small sample size. It is possible that a significant increase in serum BDNF concentrations following ECT or rTMS treatment would be observed in a larger patient population. Furthermore, the patients are heterogeneous regarding the different types of medications they were taking throughout the duration of the study. Although it was found that patients' medications in this study did not significantly affect the outcome of the results at time one or time two, different antidepressant medication may have varying effects on BDNF concentrations. Therefore the variation in medication taken by participants may have impeded our ability to detect an increase in BDNF levels. Finally, future studies should measure BDNF levels at different time points following treatment completion to report the length of time needed to observe an increase in concentration, if in fact, BDNF levels are augmented.

A further limitation in this study is the measurement of only serum BDNF levels. As it is possible that serum and cerebrospinal fluid BDNF concentrations may not be correlated (Laske et al., 2007), this study could be strengthened by measuring both serum and CSF BDNF levels. Parallel changes in BDNF levels in serum and CSF could indicate that serum BDNF reflects brain changes in BDNF following ECT and rTMS in patients with depression.
A major concern is whether or not BDNF levels measured in the blood reflect BDNF levels in the brain. Serum BDNF and whole blood BDNF levels have been positively correlated with brain tissue BDNF in rats (Karege et al., 2002b; Sartorius et al., 2009; Klein et al., 2010) and plasma BDNF has been positively correlated with hippocampal BDNF in pigs (Klein et al., 2010). However, whole blood and serum BDNF levels were negatively correlated with hippocampal BDNF levels in a genetic rat model of depression (Elfving et al., 2010). To our knowledge, no studies have investigated this relationship in humans. It remains to be determined whether BDNF concentrations in human brain tissue are reflected in plasma and serum levels.

Furthermore, Karege et al. (2005) have suggested that changes in serum BDNF levels may not be due to changes in blood BDNF concentrations themselves, but rather to changes in the release mechanisms of BDNF from platelets (Karege et al., 2005). Ninetynine percent of blood BDNF is thought to be stored and released by platelets, which are thought to sequester BDNF as opposed to synthesizing it (Karege et al., 2002b). Therefore, it is possible that platelets sequestered BDNF and were responsible for the increases in plasma BDNF following ECT and rTMS observed in previous studies, rather than an increase in BDNF synthesis. Serum BDNF measurements may not have accurately detected the true change in 


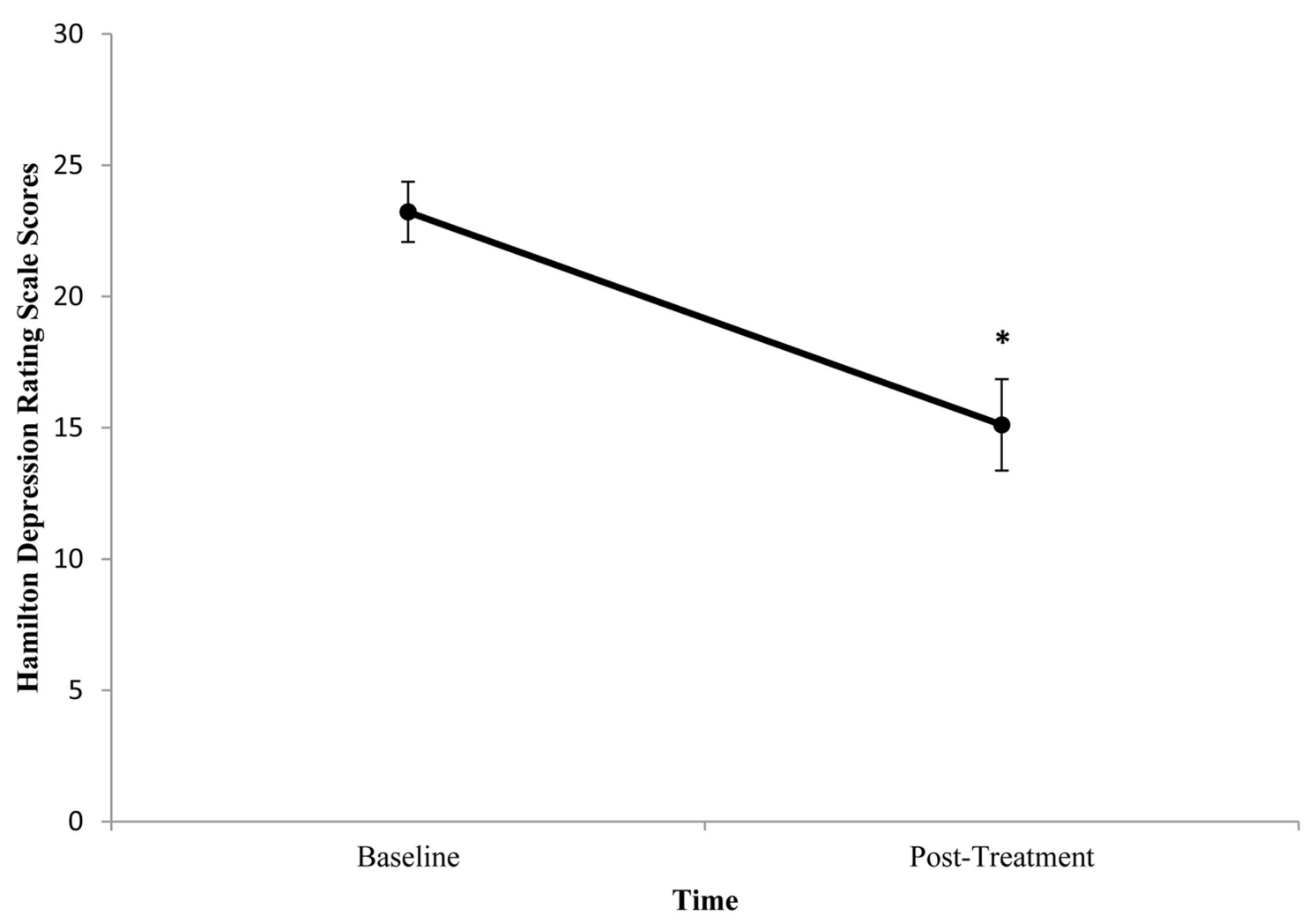

FIGURE 4 | Change in mean Hamilton Depression Rating Scale (HDRS) score from pre- to post-treatment in patients with major depressive disorder treated with repetitive transcranial magnetic stimulation
(rTMS). HDRS scores were determined 1 week prior to and 1 week following rTMS. HDRS scores significantly decreased following rTMS $(p<0.001)$. Values are mean \pm SE.
BDNF concentration, leading to a misconceived increase in BDNF levels. Therefore, it is possible that ECT and rTMS do not in fact alter serum BDNF levels in patients with MDD.

Our findings suggest that ECT and rTMS do not improve depression severity by altering serum BDNF levels. Patients in this study population were severely depressed and had undergone several trials of antidepressant treatments. As antidepressants are known to increase BDNF levels in patients with depression, it is possible that changes in serum BDNF concentrations occurred following previous pharmacological treatments and prior to the start of ECT or rTMS treatment in this study (Shimizu et al., 2003). ECT or rTMS may not further increase serum BDNF concentrations in patients who have received prior antidepressant treatment. This suggests that an increase in serum BDNF levels may occur before clinical improvement.

\section{REFERENCES}

Alderson, R. F., Alterman, A. L., Barde, Y. A., and Lindsay, R. M. (1990). Brain-derived neurotrophic factor increases survival and differentiated functions of rat septal cholinergic neurons in culture. Neuron 5, 297-306.

Altar, C. A., Whitehead, R. E., Chen, R., Wörtwein, G., and Madsen, T. M.
(2003). Effects of electroconvulsive seizures and antidepressant drugs on brain-derived neurotrophic factor protein in rat brain. Biol. Psychiatry 54, 703-709.

Angelucci, F., Aloe, L., Jiménez-Vasquez, P., and Mathé, A. (2002). Electroconvulsive stimuli alter the regional concentrations of nerve growth factor, brain-derived neurotrophic factor,

\section{CONCLUSION}

Neither ECT nor rTMS altered BDNF levels in patients with drugresistant MDD, despite improving depression severity. This pilot study suggests that ECT and rTMS may not share a similar mechanism of action as antidepressants in improving illness severity in patients with treatment-resistant depression. The role of serum BDNF concentration as a biomarker for the treatment of MDD remains to be investigated.

\section{CLINICAL TRIALS REGISTRATION \\ ClinicalTrials.gov identifier: NCT00630123.}

\section{ACKNOWLEDGMENTS}

The authors would like to thank Alan Lowe, Ann Shea, Judy Joannette, Teresa Garrah, and Liane Tackaberry.

and glial cell line-derived neurotrophic factor in adult rat brain. J. ECT 18, 138-143.

Angelucci, F., Brene, S., and Mathe, A. A. (2005). BDNF in schizophrenia, depression and corresponding animal models. Mol. Psychiatry 10, 345-352.

Aydemir, O., Deveci, A., and Taneli, F. (2005). The effect of chronic antidepressant treatment on serum brain-derived neurotrophic factor levels in depressed patients: a preliminary study. Prog. Neuropsychopharmacol. Biol. Psychiatry 29, 261-265.

Bocchio-Chiavetto, L., Bagnardi, V., Zanardini, R., Molteni, R., Nielsen, M. G., Placentino, A., and Giovannini, C. (2010). Serum and plasma 
BDNF levels in major depression: a replication study and meta-analyses. World J. Biol. Psychiatry 11, 763-773.

Bocchio-Chiavetto, L., Zanardini, R., Bortolomasi, M., Abate, M., Segala, M., Giacopuzzi, M., Riva, M. A., Marchina, E., Pasqualetti, P., Perez, J., and Gennarelli, M. (2006). Electroconvulsive therapy (ECT) increases serum brain derived neurotrophic factor (BDNF) in drug resistant patients. Eur. Neuropsychopharmacol. 16, 620-624.

Bremner, J. D., Narayan, M., Anderson, E. R., Staib, L. H., Miller, H. L., and Charney, D. S. (2000). Hippocampal volume reduction in major depression. Am. J. Psychiatry 157, 115-118.

Brunoni, A. R., Lopes, M., and Fregni, F. (2008). A systematic review and meta-analysis of clinical studies on major depression and BDNF levels: implications for the role of neuroplasticity in depression. Int. J. Neuropsychopharmacol. 11, 1169-1180.

Chen, B., Dowlatshahi, D., MacQueen, G., Wang, J., and Young, L. T. (2001). Increased hippocampal bdnf immunoreactivity in subjects treated with antidepressant medication. Biol. Psychiatry 50, 260-265.

Eaton, M. J., and Whittemore, S. R. (1996). Autocrine BDNF secretion enhances the survival and serotonergic differentiation of raphe neuronal precursor cells grafted into the adult rat CNS. Exp. Neurol. 140, 105-114.

Elfving, B., Plougmann, P. H., Müller, H. K., Mathé, A. A., Rosenberg, R., and Wegener, G. (2010). Inverse correlation of brain and blood BDNF levels in a genetic rat model of depression. Int. J. Neuropsychopharmacol. 13, 563-572.

Fernandes, B., Gama, C. S., Massuda, R., Torres, M., Camargo, D., Kunz, M., Belmonte-de-Abreu, P. S., Kapczinski, F., de Almeida Fleck, M. P., and Inês Lobato, M. (2009). Serum brain derived neurotrophic factor (BDNF) is not associated with response to electroconvulsive therapy (ECT): a pilot study in drug resistant depressed patients. $\mathrm{Neu}$ rosci. Lett. 453, 195-198.

Gershon, A. A., Dannon, P. N., and Grunhaus, L. (2003). Transcranial magnetic stimulation in the treatment of depression. Am. J. Psychiatry 160, 835-845.

Gervasoni, N., Aubry, J. M., Bondolfi, G., Osiek, C., Schwald, M., Bertschy, G., and Karege, F. (2005). Partial normalization of serum brain-derived neurotrophic factor in remitted patients after a major depressive episode. Neuropsychobiology 51, 234-238.
Ghosh, A., Carnahan, J., and Greenberg, M. E. (1994). Requirement for BDNF in activity-dependent survival of cortical neurons. Science 263,1618-1623.

Gonul, A. S., Akedeniz, F., Taneli, F., Donat, O., Eker, C., and Vahip, S. (2005). Effect of treatment on serum brain-derived neurotrophic factor levels in depressed patients. Eur. Arch. Psychiatry Clin. Neurosci. 255, 381-386.

Gronli, O., Stensland, G. Ø., Wynn, R., and Olstad, R. (2009). Neurotrophic factors in serum following ECT: a pilot study. World J. Biol. Psychiatry 10, 295-301.

Hashimoto, K., Shimizu, E., and Iyo, M. (2004). Critical role of brain-derived neurotrophic factor in mood disorders. Brain Res. Rev. 45, 104-114.

Hellweg, R., Ziegenhorn, A., Heuser, I., and Deuschle, M. (2008). Serum concentrations of nerve growth factor and brain-derived neurotrophic factor in depressed patients before and after antidepressant treatment. Pharmacopsychiatry 41, 66-71.

Hoffman, R. E., and Cavus, I. (2002). Slow transcranial magnetic stimulation, long-term depotentiation, and brain hyperexcitability disorders. Am. J. Psychiatry 159, 1093-1102.

Hu, Y., Yu, X., Yang, F., Si, T., Wang, W., Tan, Y., Zhou, D., Wang, H., and Chen, D. (2010). The level of serum brain-derived neurotrophic factor is associated with the therapeutic efficacy of modified electroconvulsive therapy in Chinese patients with depression. J. ECT 26, 121-125.

Huang, T. L., Lee, C. T., and Liu, Y. L. (2007). Serum brain-derived neurotrophic factor levels in patients with major depression: effects of antidepressants. J. Psychiatr. Res. 45, 521-525.

Hyman, C., Hofer, M., Barde, Y. A., Juhasz, M., Yancopoulos, G. D., and Squinto, S. P. (1991). BDNF is a neurotrophic factor for dopaminergic neurons of the substantia nigra. Nature 350, 230-232.

Kaplan, D. R., and Miller, F. D. (2000). Neurotrophin signal transduction in the nervous system. Curr. Opin. Neurobiol. 10, 381-391.

Karege, F., Bondolfi, G., Gervasoni, N., Schwald, M., Aubry, J. M., and Bertschy, G. (2005). Low brain derived neurotrophic factor (BDNF) levels in serum of depressed patients probably results from lowered platelet BDNF release unrelated to platelet reactivity. Biol. Psychiatry 57, 1068-1072.

Karege, F., Perret, G., Bondolfi, G., Schwald, M., Bertschy, G., and Aubry,
J. M. (2002a). Decreased serum brain-derived neurotrophic factor levels in major depressed patients. Psychiatry Res. 109, 143-148.

Karege, F., Schwald, M., and Cisse, M. (2002b). Postnatal developmental profile of brain-derived neurotrophic factor in rat brain and platelets. Neurosci. Lett. 328, 261-264.

Klein, A. B., Williamson, R., Santini, M., Clemmensen, C., Ettrup, A., Rios, M., Knudsen, G. M., and Aznar S. (2010). Blood BDNF concentrations reflect brain tissue BDNF levels across species. Int. J. Neuropsychopharmacol. 7, 1-7.

Lang, U. E., Bajbouj, M., Gallinat, J., and Hellweg, R. (2006). Brain-derived neurotrophic factor serum concentrations in depressive patients during vagus nerve stimulation and repetitive transcranial magnetic stimulation. Psychopharmacology (Berl.) 187, 56-59.

Laske, C., Stransky, E., Leyhe, T. Eschweiler, G. W., Maetzler, W., Wittorf, A., Soekadar, S., Richartz, E., Koehler, N., Bartels, M., Buchkremer, G., and Schott, K. (2007). BDNF serum and CSF concentrations in Alzheimer's disease, normal pressure hydrocephalus and healthy controls. J. Psychiatr. Res. 41, 387-394.

Lee, B. H., Kim, H., Park, S. H., and Kim, Y. K. (2007). Decreased plasma BDNF level in depressive patients. J. Affect. Disord. 101, 239-244.

Lindholm, D., Carroll, P., Tzimagiogis, G., and Thoenen, H. (1996). Autocrine-paracrine regulation of hippocampal neuron survival by IGF- 1 and the neurotrophins BDNF, NT-3 and NT-4. Eur. J. Neurosci. 8, 1452-1460.

Lisanby, S. H. (2007). Electroconvulsive therapy for depression. N. Eng. J. Med. 357, 1939-1945.

Marano, C. M., Phatak, P., Vemylapalli, U. R., Sasan, A., Nalbandyan, M. R., Ramanujam, S., Soekadar, S., Demosthenous, M., and Regenold, W. T. (2007). Increased plasma concentration of brain-derived neurotrophic factor with electroconvulsive therapy: a pilot study in patients with major depression. J. Clin. Psychiatry 68, 512-517.

Matrisciano, F., Bonaccorso, S., Ricciardi, A., Scaccianoce, S., Panaccione, I., Wang, L., Ruberto, A., Tatarelli, R., Nicoletti, F., Girardi, P., and Shelton, R. C. (2009). Changes in BDNF serum levels in patients with depression disorder (MDD) after 6 months treatment with sertraline, escitalopram, or venlafaxine. J. Psychiatr. Res. 43, 247-254.
Monteleone, P., Serritella, C., Martiadis, V., and Maj, M. (2008). Decreased levels of serum brain-derived neurotrophic factor in both depressed and euthymic patients with unipolar depression and in euthymic patients with bipolar I and II disorders. Bipolar Disord. 10, 95-100.

Okamoto, T., Yoshimura, R., IkenouchiSugita, A., Hori, H., Umene-Nakano, W., Inoue, Y., Ueda, N., and Nakamura, J. (2008). Efficacy of electroconvulsive therapy is associated with changing blood levels of homovanillic acid and brain derived neurotrophic factor (BDNF) in refractory depressed patients: a pilot study. Prog. Neuropsychopharmacol. Biol. Psychiatry 32, 1185-1190.

Piccini, A., Del Debbio, A., Medda, P., Bianchi, C., Roncaglia, I., Veltri, A., Zanello, S., Massimetti, E., Origlia, N., Domenici, L., Marazziti, D., and Dell'Osso, L. (2009). Plasma brain-derived neurotrophic factor in treatmentresistant depressed patients receiving electroconvulsive therapy. Eur. Neuropsychopharmacol. 19, 349-355.

Piccinni, A., Marazziti, D., Catena, M., Domenici, L., Del Debbio, A., Bianchi, C., Mannari, C., Martini, C., Da Pozzo, E., Schiavi, E., Mariotti, A., Roncaglia, I., Palla, A., Consoli, G., Giovannini, L., Massimetti, G., and Dell'Osso, L. (2008). Plasma and serum brain-derived neurotrophic factor (BDNF) in depressed patients during 1 year of antidepressant treatments. J. Affect. Disord. 105, 279-283.

Promega. (2009). Technical Bulletin: BDNF Emax ${ }^{\circledR I m m u n o A s s a y ~ S y s t e m . ~}$ Available at: www.promega.com/ tbs/tb257/tb257.html

Rossini, P. M., Barker, A. T., Berardelli, A., Caramia, M. D., Caruso, G., Cracco, R. Q., Dimitrijevic, M. R., Hallett, M., Katayama, Y., Lucking, C. H., Maertens de Noordhout, A. L., Marsden, C. D., Murray, N. M. F., Rothwell, J. C., Swash, M., and Tomberg, C. (1994). Non-invasive electrical and magnetic stimulation of the brain, spinal cord and roots: basic principles and procedures for routine clinical application. Report of an IFCN committee. Electroencephalogr. Clin. Neurophysiol. 91, 79-92.

Russo-Neustadt, A. A., and Chen, M. J. (2005). Brain-derived neurotrophic factor and antidepressant activity. Curr. Pharm. Des. 11, 1495-1510.

Sapolsky, R. M. (1996). Why stress is bad for your brain. Science 273 , 749-750. 
Sartorius, A., Hellweg, R., Litzke, J., Vogt, M., Dormann, C., Vollmayr, B., Danker-Hopfe, H., and Gass, P. (2009). Correlations and discrepancies between serum and brain tissue levels of neurotrophins after electroconvulsive treatment in rats. Pharmacopsychiatry 42, 270-276.

Schlaepfer, T. E., Kosel, M., and Nemeroff, C. B. (2003). Efficacy of repetitive transcranial magnetic stimulation (rTMS) in the treatment of affective disorders. $\mathrm{Neu}$ ropsychopharmacology 28, 201-205.

Schmidt, H. D., and Duman, R. S. (2010). Peripheral BDNF produces antidepressant-like effects in cellular and behavioral models. Neuropsychopharmacology 35, 2378-2391.

Sen, S., Duman, R., and Sanacora, G. (2008). Serum brain-derived neurotrophic factor, depression, and antidepressant medications: metaanalyses and implications. Biol. Psychiatry 64, 527-532.

Sheline, Y. I., Sanghavi, M., Mintun, M. A., and Gado, M. H. (1999). Depression duration but not age predicts hippocampal volume loss in medically healthy women with recurrent major depression. J. Neurosci. 19, 5034-5043.

Sheline, Y. I., Wang, P. W., Gado, M. H., Csernansky, J. G., and Vannier, M. W. (1996). Hippocampal atrophy in recurrent major depression. Proc. Natl. Acad. Sci. U.S.A. 93, 3908-3913.

Shimizu, E., Hashimoto, K., Okamura, N., Koike, K., Komatsu, N., Kumakiri, C., Nakazato, M., Watanabe, H., Shinoda, N., Okada, S., and Iyo, M. (2003). Alterations of serum levels of brain-derived neurotrophic factor (BDNF) in depressed patients with or without antidepressants. Biol. Psychiatry 54, 70-75.

Tamminga, C. A., Nemeroff, C. B., Blakely, R. D., Brady, L., Carter, C. S., Davis, K. L., Dingledine, R., Gorman, J. M., Grigoriadis, D. E., Henderson, D. C., Innis, R. B., Killen, J., Laughren, T. P., McDonald, W. M., Murphy, G. M. Jr, Paul, S. M., Rudorfer, M. V., Sausville, E., Schatzberg, A. F., Scolnick, E. M., and Suppes, T. (2002). Developing novel treatments for mood disorders: accelerating discovery. Biol. Psychiatry 52, 589-609.
Warner-Schmidt, J. L., and Duman, R. S. (2006). Hippocampal neurogenesis: opposing effects of stress and antidepressant treatment. Hippocampus 16, 239-249.

Yoshimura, R., Mitoma, M., Sugita, A., Hori, H., Okamoto, T., Umene, W., Ueda, N., and Nakamura, J. (2007). Effects of paroxetine or milnacipran on serum brain-derived neurotrophic factor in depressed patients. Prog. Neuropsychopharmacol. Biol. Psychiatry 31, 1034-1037.

Yukimasa, T., Yoshimura, R., Tamagawa, A., Uozumi, T., Shinkai, K., Ueda, N., Tsuji, S., and Nakamura, J. (2006). High frequency repetitive transcranial magnetic stimulation improves refractory depression by influencing catecholamine and brain-derived neurotrophic factors. Pharmacopsychiatry 39, 52-59.

Zanardini, R., Gazzoli, A., Ventriglia, M., Perez, J., Bignotti, S., Rossini, P. M., Gennarelli, M., and BocchioChiavetto, L. (2006). Effect of repetitive transcranial magnetic stimulation on serum brain derived neurotrophic factor in drug resistant depressed patients. J. Affect. Disord. 91, 83-86.
Conflict of Interest Statement: The authors declare that the research was conducted in the absence of any commercial or financial relationships that could be construed as a potential conflict of interest.

Received: 23 May 2011; accepted: 07 February 2012; published online: 24 February 2012.

Citation: Gedge L, Beaudoin A, Lazowski $L$, $d u$ Toit $R$, Jokic $R$ and Milev $R$ (2012) Effects of electroconvulsive therapy and repetitive transcranial magnetic stimulation on serum brain-derived neurotrophic factor levels in patients with depression. Front. Psychiatry 3:12. doi: 10.3389/fpsyt.2012.00012

This article was submitted to Frontiers in Affective Disorders and Psychosomatic Research, a specialty of Frontiers in Psychiatry.

Copyright (c) 2012 Gedge, Beaudoin, Lazowski, du Toit, Jokic and Milev. This is an open-access article distributed under the terms of the Creative Commons Attribution Non Commercial License, which permits non-commercial use, distribution, and reproduction in other forums, provided the original authors and source are credited. 